\title{
Estudio del Efecto de un Enjuagatorio de Rosmarinus officinalis (Romero) en la Cicatrización de Heridas de Mucosa Oral
}

\author{
Study of the Effect of Rosmarinus officinalis (Rosemary) \\ Mouthrinse on Healing Wounds of the Oral Mucosa
}

\author{
Bestagno, V. ; Neira, I. ${ }^{1}$; Gómez-Gaete, P.; Pastene, E.. ; Gómez, M. ${ }^{3}$; Pérez, M. A. ${ }^{4}$ \& Rojas I. G. ${ }^{5}$
}

BESTAGNO, V.; NEIRA, I.; GÓMEZ-GAETE, P.; PASTENE, E.; GÓMEZ, M.; PÉREZ, M. A. \& ROJAS, I. G. Estudio del efecto de un enjuagatorio de Rosmarinus officinalis (romero) en la cicatrización de heridas de mucosa oral. Int. J. Odontostomat., 11(4):411-417, 2017.

RESUMEN: El objetivo de este estudio fue evaluar el efecto de un enjuague oral de extracto acuoso de Rosmarinus Officinalis (romero) sobre la cicatrización de heridas de mucosa oral en sujetos sanos. Veintiocho estudiantes, de sexo masculino, edad promedio de 22 años, fueron reclutados para un estudio de doble ciego en la Facultad de Odontología de la Universidad de Concepción, Chile. Se creó una herida estándar de $3 \mathrm{~mm}$ de diámetro en la mucosa palatina, que fue fotografiada durante un período de 21 días para determinar su tamaño por fotoplanimetría. Los voluntarios recibieron enjuagues orales para ser utilizados a diario desde el día de creación de la herida, 3 veces al día, y fueron divididos en grupo control (enjuague placebo) y grupo experimental (enjuague de extracto de romero). El extracto acuoso de Romero fue analizado por HPLC, mostrando un alto contenido de carnosol (83 \%) y ácido rosmarínico (65\%). Se observó una disminución significativa en el tamaño de la herida del grupo tratado con enjuague de romero comparado con el tratado con enjuague placebo al día 3 de cicatrización ( $20 \%, p=0,046$, Mann-Whitney). Posteriormente no se encontraron diferencias, completándose la cicatrización antes del día 21 para ambos grupos. Los resultados sugieren que el enjuagatorio de extracto acuoso de romero favorece la cicatrización temprana de las heridas, lo que puede deberse a su alto contenido de carnosol y ácido rosmarínico que han demostrado estimular la cicatrización y poseer actividad antimicrobiana.

PALABRAS CLAVE: cicatrización, romero, Rosemarinus officinalis, mucosa oral, enjuague.

\section{INTRODUCCIÓN}

Las heridas quirúrgicas que afectan a la mucosa de la cavidad oral son relativamente frecuentes, ya sea derivadas de procedimientos quirúrgicos como injertos autólogos de paladar, cirugía periodontal y maxilofacial y/o colocación de implantes (Zhu et al., 2015). La velocidad de cicatrización de los tejidos blandos va a depender de diversos factores que incluyen tamaño y ubicación de la herida, higiene oral y la calidad de la respuesta inmune (Zhu et al.).

El proceso de cicatrización involucra tres fases que están altamente interrelacionadas, incluyendo la fase inflamatoria, fase de formación de tejidos y una larga fase de remodelación (Martin, 1997; Singer \& Clark, 1999). La fase inflamatoria, que ocurre durante los primeros tres días de ocurrida la herida, tiene como objetivo contener la hemorragia, y reclutar células fagocíticas, como neutrófilos y macrófagos, que eliminarán los microorganismos presentes en la herida. La fase de formación de tejidos (días 3 a 10 de creación de la herida) involucra la activación de la migración y proliferación de células epiteliales, endoteliales y fibroblastos, con el objetivo de estimular la queratinización de la herida, la formación de nuevos vasos sanguíneos y el depósito de nueva matriz extracelular. Finalmente la fase de remodelación

\footnotetext{
${ }^{1}$ Departamento de Estomatología Quirúrgica, Facultad de Odontología, Universidad de Concepción, Concepción, Chile.

${ }^{2}$ Departamento de Farmacia, Facultad de Farmacia, Universidad de Concepción, Chile.

${ }^{3}$ Departamento de Odontología restauradora, facultad de Odontología, Universidad de Concepción, Chile.

${ }^{4}$ Departamento de Pediatría, Facultad de Odontología, Universidad de Concepción, Chile.

${ }^{5}$ Departament of Periodontics and Endodontics, School of Dental Medicine, State University of New York at Buffalo, Buffalo, NY, USA.
} 
involucra la maduración de los nuevos tejidos formados, y puede extenderse desde meses a años. Una fase inflamatoria insuficiente, aumentada o prolongada en el tiempo puede provocar el retraso del cierre de la herida y aumentar el riesgo de infección (Singer \& Clark; Guo \& DiPietro, 2010).

Aunque es reconocido que la mucosa oral tiene una gran capacidad de regeneración, diversos factores pueden retrasar el proceso de cierre de heridas orales, entre los cuales la infección es el más frecuente (Glim et al., 2013). Es por ello que la utilización de productos exógenos con capacidad antimicrobiana y que puedan acelerar el proceso de cierre de las heridas de mucosa oral puede ser de gran beneficio para prevenir infecciones. Dentro de la farmacopea europea, el Romero (Rosmarinus officinalis L.), especie introducida en Chile perteneciente a la familia Lamiaceae, se destaca por sus propiedades antimicrobianas, anti-inflamatorias y estimulantes de la cicatrización (Bandara et al., 2007; Bernardes et al., 2010; Yilanci et al., 2015; de Oliveira et al., 2017). Sin embargo, según nuestro conocimiento, su efecto sobre la cicatrización de heridas de mucosa oral no ha sido determinado clínicamente.

El objetivo de nuestro estudio fue evaluar el efecto de un enjuagatorio preparado en base a un extracto acuoso de romero, sobre el cierre de heridas creadas en la mucosa palatina de pacientes sanos, controlados por edad, sexo y hábitos.

\section{MATERIAL Y MÉTODO}

Participantes. Veintiocho estudiantes de Odontología, sexo masculino, edad promedio de $22,1 \pm 1,7$ años, fueron reclutados en la Facultad de Odontología de la Universidad de Concepción para participar en el estudio de doble ciego en forma voluntaria. El Comité de Ética de la Universidad de Concepción dio su aprobación institucional, y todos los participantes firmaron un consentimiento informado. Sólo fueron incluidos en el estudio los sujetos que no habían consumido antibióticos en los últimos 6 meses, no fumadores y que estaban libres de enfermedades sistémicas. Previo a la creación de la herida, cada participante fue sometido a los siguientes exámenes de laboratorio: Hemograma, Tiempo de protrombina, TTPA y Glicemia, los cuales debían ser normales para ser incluidos en el estudio. Durante la duración del estudio los participantes no podían consumir analgésicos.
Creación y monitoreo de la herida palatina. El paladar duro fue anestesiado con técnica infiltrativa terminal utilizando mepivacaína al $2 \%$ con epinefrina (1:100.000) y una herida de $3 \mathrm{~mm}$ de diámetro fue demarcada con un sacabocado para biopsias (Miltex Medical, Plainsboro, NJ; USA) entre las raíces palatinas del primer y segundo molar. Se utilizó un bisturí para remover el epitelio superficial y tejido conectivo subyacente a una profundidad de $1,5 \mathrm{~mm}$ de espesor. La herida fue comprimida por media hora con una gasa, y los sujetos fueron instruidos de realizar su higiene oral diaria complementada con el enjuagatorio que se les entregó tres veces al día hasta finalizar el estudio.

De acuerdo a lo descrito previamente (Marucha et al., 1998) la herida fue videofotografiado con una cámara intraoral (Beijing Returning Swans Technology, Beijing, China) a la media hora de su creación (día 0) y día por medio desde los días 1 al 12. Para compensar por diferencias en angulación y magnificación, una etiqueta de tamaño estándar ( $3 \mathrm{~mm}$ de diámetro) fue colocada junto a la herida al momento de su videocaptura. Las imágenes fueron digitalizadas y codificadas y se midió tanto el tamaño de la herida como de la etiqueta estándar, para obtener el tamaño normalizado de la herida. Este valor a su vez fue ajustado al tamaño original de la herida (día 0) para obtener la proporción entre el tamaño diario y el tamaño original. Tanto los investigadores que fotografiaron las heridas como el investigador que midió las heridas desconocían el tratamiento asignado a cada sujeto.

Los días siguientes al día 12, las heridas sólo fueron monitoreadas visualmente ya que no era posible distinguir mediante videofotografía el tamaño de la herida debido a la re-epitelización de ésta. Todas las heridas sanaron sin complicaciones antes del día 21. La percepción de dolor, mediante una escala visual (EVA), también fue monitoreada hasta el término del estudio.

Obtención de extracto de Romero y preparación del enjuagatorio. Para la extracción a escala del laboratorio, a $400 \mathrm{~g}$ de muestra se le agregaron 1200 $\mathrm{mL}$ de agua destilada a $80^{\circ} \mathrm{C}$ con agitación manual. Una vez alcanzados los $60-65^{\circ} \mathrm{C}$, la mezcla fue homogenizada con Ultraturrax a 20.000 rpm y el extracto acuoso fue obtenido por maceración durante 30 min con posterior separación de los sólidos insolubles mediante centrifugación (5000 rpm, $20 \mathrm{~min}$ ). El proceso se repitió dos veces con igual cantidad de agua y los tres líquidos resultantes fueron reunidos. La purificación final y obtención del extracto acuoso de rome- 
ro se realizó de acuerdo al procedimiento publicado por Pastene et al. (2009). El polvo de extracto de romero (EROM) fue almacenado en desecador bajo vacío durante 24-48 h para luego ser envasado en un recipiente ámbar y guardado a $-80{ }^{\circ} \mathrm{C}$ hasta el momento de su empleo. El EROM fue analizado por RPHPLC, usando el método publicado por Pastene et al. (2010). La determinación de polifenoles totales en el extracto de romero se llevó a cabo por el método FolinCiocalteu (Singleton \& Rossi, 1965) con algunas modificaciones. Se utilizó como estándar el ácido gálico con el que se preparó una curva de calibración.

El enjuague de Romero fue preparado a partir del EROM a una concentración de 1,5 g/L en agua nanopura y sometido a filtración bacteriológica con un sistema de filtración desechable (Whatman Klari-Flex, Sigma, St. Louis, MO, USA) $(\mathrm{pH}=5.23)$. El enjuague placebo fue preparado con agua destilada recién hervida, la cual fue sometida al proceso de filtración previamente mencionado $(\mathrm{pH}=5.57)$.

Tratamiento con Enjuague de Romero. Para el tratamiento con enjuague, el grupo de 28 pacientes fue asignado aleatoriamente, por un colaborador independiente, al uso de enjuagatorio con extracto de romero o placebo. Los sujetos debían utilizar el enjuague por 30 segundos, tres veces al día durante la duración del estudio, y mantenerlo refrigerado. Cada 5 días se les entregó un nuevo enjuague para evitar contaminación microbiana. Durante la duración del estudio muestras del enjuague utilizado fueron incubados a $37^{\circ} \mathrm{C}$ para detectar crecimiento microbiano, el cual fue negativo.

Análisis estadístico. Se obtuvo la proporción entre el tamaño normalizado de la herida (días 1 al 12) y la herida inicial (día 0) para cada participante. Se utilizó el test $U$ de Mann-Whitney para determinar diferencias estadísticamente significativas en el tamaño de la herida entre el grupo tratado con enjuague placebo y el grupo tratado con enjuague de Romero. Los datos fueron expresados como promedio y error estándar, у $\mathrm{p}<0.05$ (two-tailed) fue considerado estadísticamente significativo.

\section{RESULTADOS}

El análisis del extracto de romero (EROM) usando el método de Folin-Ciocalteau dio como resultado un $38,6 \%$ de polifenoles totales expresados como equivalente de ácido gálico (EAG). El análisis de los marcadores químicos del romero mediante HPLC es resumido en la Tabla I, donde se destaca el alto contenido del extracto en carnosol $(83,67 \%)$ y ácido rosmarínico $(65,22 \%)$.

Tabla I. Composición del extracto acuoso de Romero medido mediante HPLC.

\begin{tabular}{lc}
\hline Compuesto & Concentración $(\mathrm{mg} / \mathrm{g})$ \\
\hline Ácido rosmarínico & $\mathbf{6 5 , 2 2} \pm \mathbf{0 , 1 6}$ \\
Luteolina & $40,07 \pm 0,12$ \\
Ácido cafeico & $31,25 \pm 0,07$ \\
Apigenina & $27,08 \pm 0,19$ \\
Rosmanol & $1,15 \pm 0,05$ \\
Cirsimaritina & $0,12 \pm 0,15$ \\
Carnosol & $\mathbf{8 3 , 6 7 \pm 0 , 1 1}$ \\
Ácido carnósico & $24,12 \pm 0,09$ \\
\hline
\end{tabular}

De los 28 sujetos incluidos en el estudio, tres debieron ser eliminados debido al consumo de analgésicos o por no seguir las instrucciones del estudio. Los grupos quedaron conformados por 11 sujetos tratados con enjuague placebo y 14 sujetos con enjuague de extracto de romero. No se observaron diferencias demográficas ni de hábitos entre ambos grupos. Tampoco hubo diferencias en la percepción de dolor (escala EVA) entre grupo experimental y placebo, el cual fue mínimo y limitado al primer día. Los enjuagues placebo y de romero no mostraron crecimiento microbiano durante la duración del estudio al ser incubados a $37^{\circ} \mathrm{C}$.

La Figura 1 muestra heridas representativas de los grupos tratados con enjuague placebo y de extracto de romero los días 0,3 y 12 de cicatrización. El análisis del tamaño de las heridas en relación a su tamaño original (día 0), mostró que al día 1, ambos grupos tuvieron un aumento en el tamaño promedio de las heridas en relación al día 0 de alrededor del 25 \% (Fig. 2). Posterior al día 1 las heridas comenzaron a cerrar, alcanzando el $70 \%$ de cierre al día 12 (Fig. 2). Se observó que el día 3 de cicatrización hubo una disminución del $20 \%$ en el tamaño de las heridas en el grupo tratado con enjuague de romero (promedio $=0,92 \pm 0,07$, mediana $=0,93$ ) comparado con el grupo tratado con enjuague placebo (promedio $=1,170 \pm 0,11$, mediana $=1,19)(p=0,046$, MannWhitney) (Fig. 2). En la Figura 3 se muestra la distribución del tamaño de las heridas (en relación al tamaño original) en el día 3 de cada uno de los sujetos en los grupos tratados con enjuague placebo y de extracto de romero, donde se observan diferencias significativas. No se observaron diferencias estadísticamente significativas entre los grupos en el resto de los días del proceso de cicatrización. Todas las heridas se encontraron cerradas al día 21 del estudio. 

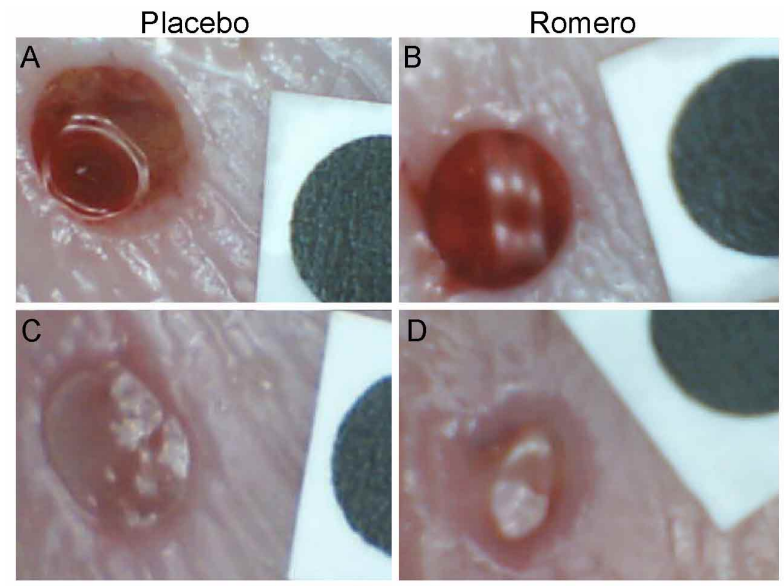

Día 0
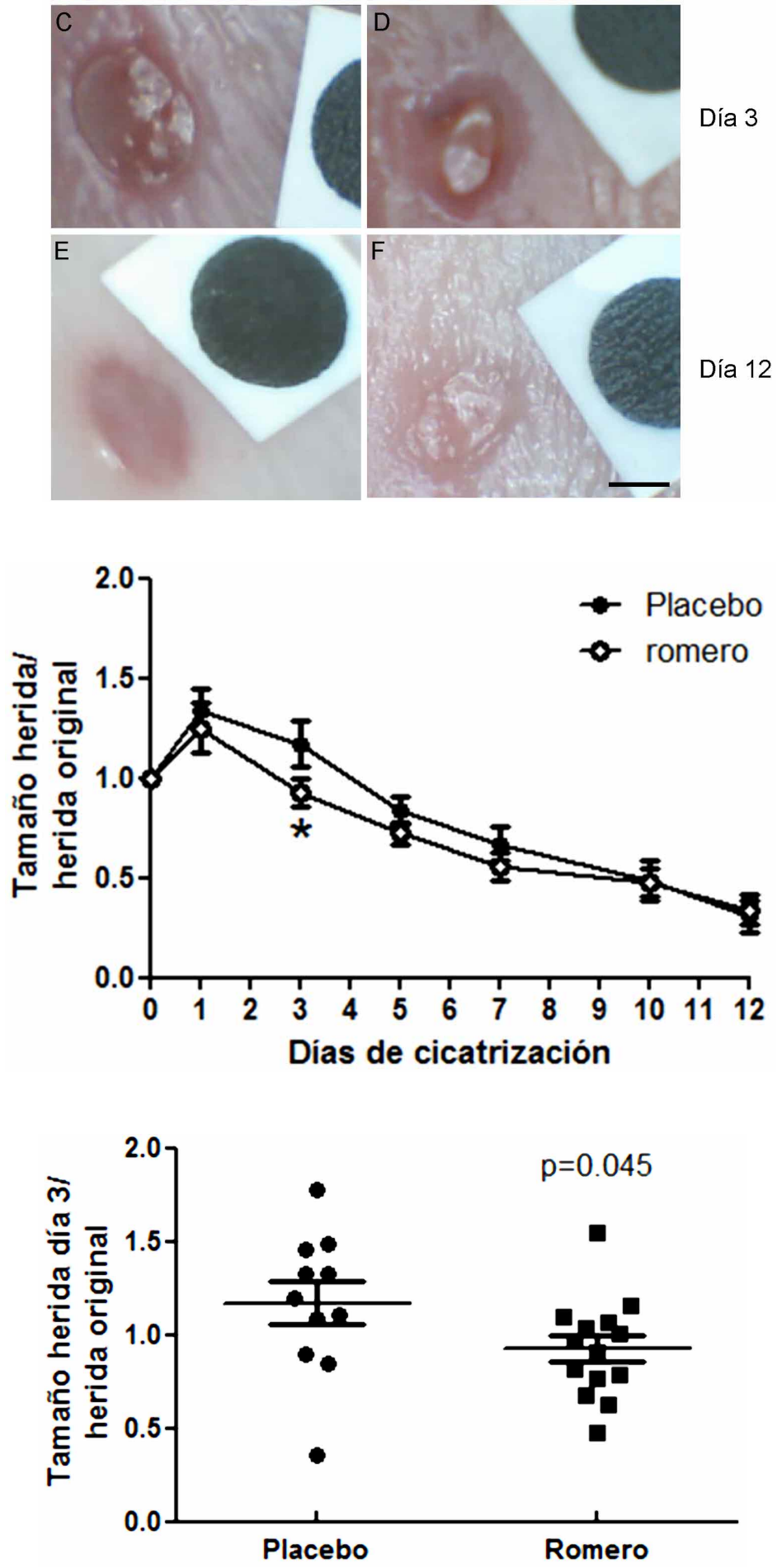

Fig. 1. Fotografías representativas del monitoreo del efecto del enjuague de extracto acuoso de romero y de enjuague placebo en la cicatrización de heridas de mucosa oral. Imágenes muestran heridas representativas en el día $0(A, B)$, día $3(C, D)$ y día $12(E, F)$, en los grupos tratados con enjuague placebo ( $A, C$ y $E$ ) y enjuague de extracto de romero $(B, D$ y $F)$. Las imágenes fueron obtenidas mediante videofotografía, y se utilizó una etiqueta estándar de $3 \mathrm{~mm}$ de diámetro para obtener el tamaño normalizado de las heridas para su análisis por fotoplanimetría. La barra representa $1 \mathrm{~mm}$.

Día 12
Fig. 2. Efecto del extracto acuoso de romero sobre la cinética de cierre de heridas de mucosa oral. Las heridas en mucosa de paladar fueron creadas con un sacabocado de $3 \mathrm{~mm}$ y videofotografiadas desde el día 0 al 12. Las imágenes fueron digitalizadas y analizadas para determinar la proporción entre el tamaño diario de la herida en relación al tamaño original de la herida (día 0). * $p<0.05$ (Mann-Whitney) para el día 3 entre el grupo tratado con enjuague de Romero y el grupo tratado con enjuague placebo.

Fig. 3. Distribución individual del tamaño de las heridas en el día 3 de cicatrización en el grupo tratado con enjuague de Romero y el grupo tratado con enjuague placebo. El tamaño de las heridas fue obtenido por fotoplanimetría, como se detalló en Métodos. ${ }^{*} p=0.046$ (Mann-Whitney) para el promedio del tamaño de las heridas del grupo tratado con enjuague de Romero comparado con el tamaño promedio de las heridas del grupo tratado con enjuague placebo. 
BESTAGNO, V.; NEIRA, I.; GóMEZ-GAETE, P.; PASTENE, E.; GóMEZ, M.; PÉREZ, M. A. \& ROJAS, I. G. Estudio del efecto de un enjuagatorio de Rosmarinus officinalis (romero) en la cicatrización de heridas de mucosa oral. Int. J. Odontostomat., 11(4):411-417, 2017.

\section{DISCUSIÓN}

El presente estudio mostró una reducción significativa (20\%) en el cierre inicial (día 3 ) de heridas de mucosa palatina de pacientes sanos tratados con enjuague de extracto acuoso de romero, al comparar con enjuague placebo. Este efecto sólo se observó en la etapa temprana de cicatrización donde predominan las respuestas inflamatoria y antimicrobiana (Martin).

La etapa inflamatoria de cicatrización (días 13 ), se caracteriza por el reclutamiento de células fagocíticas (neutrófilos y macrófagos) cuya principal función es tanto para eliminar a los microorganismos que pueden estar infectando la herida, como para preparar los tejidos para la reparación (Singer \& Clark). Los resultados sugieren que el romero tendría un efecto antimicrobiano y modulador de la respuesta inflamatoria que permitiría un cierre inicial más acelerado de la herida. Este efecto del enjuague de romero puede ser relevante para el cuidado post-quirúrgico en intervenciones en pacientes con cicatrización disminuida, ya sea por edad avanzada, uso de medicamentos inmunosupresores (ej. corticoides, terapia inmunosupresora en pacientes trasplantados), por enfermedades sistémicas como la diabetes, o por tabaquismo (Guo \& DiPietro; Smith et al., 2015).

El romero (Rosmarinus officinalis L.) es una especie introducida en Chile perteneciente a la familia Lamiaceae, la cual está constituida por unas 3.000 especies que crecen y se desarrollan en diferentes hábitats. El romero es un arbusto de hojas coriáceas y lineales que florece prácticamente todo el año. Tradicionalmente, el romero se ha utilizado a nivel sistémico como agente colagogo y colerético (estimula la producción de bilis) y también diurético (Bandara et al.). Las referencias al uso externo del romero incluyen las compresas para el reumatismo, neuralgias y alopecia (Hay et al., 1998; Lukaczer et al., 2005).

Químicamente, el romero se caracteriza por su alto contenido en aceites esenciales (1-2,5\%), entre los cuales predominan el alcanfor (15-25\%), cineol (15-30\%), -pineno (25\%) y borneol (Atti-Santos et al., 2005; Zaouali et al., 2010), los que han mostrado propiedades antibacterianas y antimicóticas contra patógenos comunes de la cavidad oral (de Oliveira et al.). Dentro de la fracción no volátil, se ha informado de la presencia de al menos 15 flavonoides, con estructuras diversas derivadas de luteolina, kaempferol, genkwanina, diosmina, entre otros (Ho et al., 2000;
Bai et al., 2010). Otro grupo de compuestos ampliamente estudiado son los derivados del ácido cafeico, como el ácido rosmarínico, y diterpenos tricíclicos, entre los que destacan el rosmaridifenol, rosmanol, rosmanial, rosmarisina, carnosol y ácido carnósico (Bruneton, 1995). El romero también posee los triterpenos pentacíclicos, ácidos ursólico y oleanólico, los que poseen propiedades antimicrobianas (Horiuchi et al., 2007), citotóxicas (Liu, 1995, 2005), antiinflamatorias y antioxidantes (Najid et al., 1992; Kapil \& Sharma, 1995). Tanto los aceites esenciales como el extracto acuoso del romero han mostrado efectos benéficos similares en la estimulación de la cicatrización de heridas (Abu-Al-Basal, 2010).

En este estudio, el análisis del extracto acuoso de romero obtenido para crear el enjuague oral mostró una alta concentración de ácido rosmarínico $(65$ $\%$ ) y de carnosol (83\%), seguido por luteolina (40\%), ácido cafeico (31\%), apigenina $(27 \%)$ y ácido carnósico (24\%), y bajas concentraciones de rosmanol y cirsimaritina ( $\leq 1 \%)$. Recientemente, se ha reportado que la aplicación tópica del ácido rosmarínico favorece la cicatrización de heridas cutáneas en un modelo animal de ratas Wistar (Yilanci et al.). Por otra parte, los componentes carnosol y ácido carnósico han mostrado un efecto antimicrobiano sobre bacterias comunes del biofilm oral como Streptococcus mutans, S. salivarius, S. sobrinus, S. mitis, S. sanguinis, y Enterococcus faecalis (Bernardes et al.). Jordán et al. (2012) mostró que la concentración de carnosol es de gran importancia para la actividad antimicrobiana de los extractos de romero. Más aún, la combinación del carnosol con antibióticos puede potenciar el efecto antimicrobiano, particularmente contra bacterias resistentes a varios antibióticos (Jarrar et al., 2010; Jordán et al.).

El uso de enjuagues con propiedades antimicrobianas y cicatrizantes es común en odontología para el cuidado post-quirúrgico, y aunque existen una serie de preparaciones disponibles en el mercado éstas aún poseen una serie de limitaciones (Salmerón Escobar \& del Amo Fernández de Velasco, 2010). Los resultados de nuestro estudio sugieren que el enjuague de romero en base a su extracto acuoso puede favorecer la etapa inicial de la cicatrización, la cual es crítica para el control de la infección y el inicio de la reparación. Futuros estudios deberán enfocarse en evaluar el efecto de la utilización conjunta de aceites esenciales y extracto acuoso de romero en enjuagues orales, así como también el efecto del romero en pacientes con cicatrización disminuida que son cada vez más abundantes en nuestra población. 
AGRADECIMIENTOS. Este estudio fue financiado por proyectos DIUC No 212.101.007-1.0 (IP Dr. Vally Bestagno) De la Vicerrectoría de Investigación de la Universidad de Concepción y por el proyecto Fondecyt 1090287 (IP Dr. IG Rojas), CONICYT Chile.

BESTAGNO, V.; NEIRA, I.; GÓMEZ-GAETE, P.; PASTENE, E.; GÓMEZ, M.; PÉREZ, M. A. \& ROJAS, I. G. Study of the effect of Rosmarinus officinalis (Rosemary) mouthrinse on healing wounds of the oral mucosa. Int. J. Odontostomat., 11(4):411-417, 2017.

ABSTRACT: The objective of the study was to evaluate the effect of a mouthrinse containing an aqueous extract of Rosmarinus officinalis (rosemary) on healing wounds of the oral mucosa in healthy subjects. Twenty Eight healthy, non-smoking male students, mean age 22 years, were recruited for a double-blind study at the School of Dentistry of the University of Concepción, Chile. A standard $3 \mathrm{~mm}$ wound was created on the palate with a punch biopsy. The wounds were photographed to assess healing for 21 days. The subjects were instructed to use a mouthrinse 3 times daily for 21 days, starting the day of wounding, and were divided into a control group (placebo) and an experimental group (rosemary extract). The aqueous rosemary extract used for the mouthrinse had a high content of carnosol (83\%) and rosmarinic acid ( $65 \%$ ) as determined by HPLC. We observed a $20 \%$ reduction in wound size by day 3 in the rosemary mouthrinse group as compared to the placebo mouthrinse group ( $p=0.04$, Mann Whitney). Thereafter, no significant differences in wound closure were observed. Healing was completed by day 21 in both groups. The results suggest that the mouthrinse containing the aqueous rosemary extract stimulates early healing of mucosal wounds. This could be due to the high content of carnosol and rosmarinic acid, which are known for their healing and antimicrobial properties.

KEY WORDS: rosemary, Rosemarinus officinalis, wound healing, oral mucosa, mouthrinse.

\section{REFERENCIAS BILIOGRÁFICAS}

Abu-Al-Basal, M. A. Healing potential of Rosmarinus officinalis $L$. on full-thickness excision cutaneous wounds in alloxan-induced-diabetic BALB/c mice. J. Ethnopharmacol., 131(2):443-50, 2010.

Atti-Santos, A. C.; Rossato, M.; Pauletti, G. F.; Rota, L. D.; Rech, J. C.; Pansera, M. R.; Agostini, F.; Serafini, L. A. \& Moyna, P. Physico-chemical evaluation of Rosmarinus officinalis L. essential oils. Braz. Arch. Biol. Technol., 48(6):1035-9, 2005.

Bai, N.; He, K.; Roller, M.; Lai, C. S.; Shao, X.; Pan, M. H. \& Ho, C. T. Flavonoids and phenolic compounds from Rosmarinus officinalis. J. Agric. Food Chem., 58(9):53637, 2010.
Bandara, M. S.; Tanino, K. K. \& Acharya, S. N. Rosemary (Rosmarinus officinalis L.): A Medicinal Plant Species. In: Acharya, S. N. \& Thomas, J. E. (Eds.). Advances in Medicinal Plant Research. Trivandrum, Research Signpost, 2007. pp.173-94.

Bernardes, W. A.; Lucarini, R.; Tozatti, M. G.; Souza, M. G.; Silva, M. L.; Filho, A. A.; Martins, C. H.; Crotti, A. E.; Pauletti, P. M.; Groppo, M. \& Cunha, W. R. Antimicrobial activity of Rosmarinus officinalis against oral pathogens: relevance of carnosic acid and carnosol. Chem. Biodivers., 7(7):1835-40, 2010.

Bruneton, J. Pharmacognosy, Phytochemistry, Medicinal Plants. Andover, Intercept Ltd., 1995.

de Oliveira, J. R.; de Jesus, D.; Figueira, L. W.; de Oliveira, F. E.; Pacheco Soares, C.; Camargo, S. E.; Jorge, A. O. \& de Oliveira, L. D. Biological activities of Rosmarinus officinalis L. (rosemary) extract as analyzed in microorganisms and cells. Exp. Biol. Med. (Maywood), 242(6):625-34, 2017.

Glim, J. E.; van Egmond, M.; Niessen, F. B.; Everts, V. \& Beelen, R. H. Detrimental dermal wound healing: what can we learn from the oral mucosa? Wound Repair Regen., 21(5):648-60, 2013.

Guo, S. A. \& DiPietro, L. A. Factors affecting wound healing. J. Dent. Res., 89(3):219-29, 2010.

Hay, I. C.; Jamieson, M. \& Ormerod, A. D. Randomized trial of aromatherapy. Successful treatment for alopecia areata. Arch. Dermatol., 134(11):1349-52, 1998.

Ho, C. T.; Wang, M.; Wei, G. J.; Huang, T. C. \& Huang, M. T. Chemistry and antioxidative factors in rosemary and sage. Biofactors, 13(1-4):161-6, 2000.

Horiuchi, K.; Shiota, S.; Hatano, T.; Yoshida, T.; Kuroda, T. \& Tsuchiya, T. Antimicrobial Activity of Oleanolic Acid from Salvia officinalis and Related Compounds on VancomycinResistant Enterococci (VRE). Biol. Pharm. Bull., 30(6):1147-9, 2007.

Jarrar, N.; Abu-Hijleh, A. \& Adwan, K. Antibacterial activity of Rosmarinus officinalis L. alone and in combination with cefuroxime against methicillin-resistant Staphylococcus aureus. Asian Pac. J. Trop. Med., 3(2):121-3, 2010.

Jordán, M. J.; Lax, V.; Rota, M. C.; Lorán, S. \& Sotomayor, J. A. Relevance of carnosic acid, carnosol, and rosmarinic acid concentrations in the in vitro antioxidant and antimicrobial activities of Rosmarinus officinalis (L.) methanolic extracts. J. Agric. Food Chem., 60(38):96038, 2012

Kapil, A. \& Sharma, S. Effect of oleanolic acid on complement in adjuvant- and carrageenan-induced inflammation in rats. J. Pharm. Pharmacol., 47(7):585-7, 1995.

Liu, J. Oleanolic acid and ursolic acid: research perspectives. J. Ethnopharmacol., 100(1-2):92-4, 2005.

Liu, J. Pharmacology of oleanolic acid and ursolic acid. J. Ethnopharmacol., 49(2):57-68, 1995.

Lukaczer, D.; Darland, G.; Tripp, M.; Liska, D.; Lerman, R. H.; Schiltz, B. \& Bland, J. S. A pilot trial evaluating Meta050, a proprietary combination of reduced iso-alpha acids, rosemary extract and oleanolic acid in patients with arthritis and fibromyalgia. Phytother. Res., 19(10):864-9, 2005. 
BestAGnO, V.; NEIRA, I.; GómEZ-GAETE, P.; PASTENE, E.; GóMEZ, M.; PÉREZ, M. A. \& RoJAS, I. G. Estudio del efecto de un enjuagatorio de Rosmarinus officinalis (romero) en la cicatrización de heridas de mucosa oral. Int. J. Odontostomat., 11(4):411-417, 2017.

Martin, P. Wound healing--aiming for perfect skin regeneration. Science, 276(5309):75-81, 1997.

Marucha, P. T.; Kiecolt-Glaser, J. K. \& Favagehi, M. Mucosal wound healing is impaired by examination stress. Psychosom. Med., 60(3):362-5, 1998.

Najid, A.; Simon, A.; Cook, J.; Chable-Rabinovitch, H.; Delage, C.; Chulia, A. J. \& Rigaud, M. Characterization of ursolic acid as a lipooxygenase and cyclooxygenase inhibitor using macrophages, platelets, anddifferentiated HL60 leukemic cells. FEBS Lett., 299(3):213-7, 1992.

Pastene, E.; Speisky, H.; García, A.; Moreno, J.; Troncoso, M. \& Figueroa, G. In vitro and in vivo effects of apple peel polyphenols against Helicobacter pylori. J. Agric. Food Chem., 58(12):7172-9, 2010.

Pastene, E.; Troncoso, M.; Figueroa, G.; Alarcón, J. \& Speisky, H. Association between polymerization degree of apple peel polyphenols and inhibition of Helicobacter pylori urease. J. Agric. Food Chem., 57(2):416-24, 2009.

Salmerón Escobar, J. I. \& del Amo Fernández de Velasco, A. Profilaxis antibiótica en cirugía oral y maxilofacial. Med. Oral Patol. Oral Cir. Oral, 11(3):292-6, 2006.

Singer, A. J. \& Clark, R. A. Cutaneous wound healing. N. Engl. J. Med., 341(10):738-46, 1999.

Singleton, V. L. \& Rossi, J. A. Colorimetry of total phenolics with phosphomolybdic-phosphotungstic acid reagents. Am. J. Enol. Vitic., 16(3):144-58, 1965.

Smith, P. C.; Cáceres, M.; Martínez, C.; Oyarzún, A. \& Martínez, J. Gingival wound healing: an essential response disturbed by aging? J. Dent. Res., 94(3):395402, 2015.

Yilanci, S.; Bali, Y. Y.; Yuzbasioglu, M.; Unlu, R. E.; Orhan, E.; Simon, A.; Tóth, G.; Demirezer, L. O. \& KuruuzumUz, A. The evaluation of wound healing potential of rosmarinic acid isolated from Arnebia purpurea. Planta Med., 81(16):135, 2015.

Zaouali, Y.; Bouzaine, T. \& Boussaid, M. Essential oils composition in two Rosmarinus officinalis L. varieties and incidence for antimicrobial and antioxidant activities. Food Chem. Toxicol., 48(11):3144-52, 2010.

Zhu, T.; Park, H. C.; Son, K. M. \& Yang, H. C. Effects of dimethyloxalylglycine on wound healing of palatal mucosa in a rat model. BMC Oral Health, 15:60, 2015.
Dirección para correspondencia

Isolde Gina Rojas-Rudolph

Department of Periodontics and Endodontics

250 Squire Hall

School of Dental Medicine

University at Buffalo

Buffalo

New York

USA

Email: isolde.gina@gmail.com isoldero@buffalo.edu

Recibido : 14-07-2017

Aceptado: 13-09-2017 